\title{
Determination of the Left Ventricular Asynergic Site by QRST Isointegral Mapping in Patients with Myocardial Infarction
}

\author{
Isao Kubota, M.D.,* Kozue Ikeda, M.D.,* Michiyasu Yamaki, M.D.,* \\ Yoshihiko Watanabe, M.D., ${ }^{* *}$ Kai Tsuiki, M.D., ${ }^{*}$ \\ and Shoji YAsur, M.D.*
}

\section{SUMMARY}

QRST isointegral maps were made from 87 body surface ECGs in 41 patients with prior myocardial infarction. To evaluate the abnormalities of QRST isointegral maps, a deviation map was constructed from a deviation index, which expressed the deviation of the QRST time-integral value from the normal value at each lead point. Patients were divided into 3 groups according to the asynergic site on left ventriculograms: patients with anterior wall asynergy $(n=13)$, those with inferior wall asynergy $(n=13)$ and those with both anterior and inferior asynergies $(n=15)$. A consistent deviation map pattern was obtained in each group regardless of the QRS duration. Consequently, the deviation map was considered to be a practical method of identifying the asynergic site in patients with myocardial infarction, especially in those with intraventricular conduction disturbances. It is suggested that the deviation map can reveal abnormally altered intrinsic ventricular recovery properties resulting from a loss of the electromotive force of the infarcted myocardium.

\section{Additional Indexing Words:}

Body surface mapping Deviation map Intraventricular conduction disturbances

R ECENTLY, isointegral analysis of body surface potential maps was pro$\mathbf{Z}$ posed by Montague et al as a useful approach to data reduction. ${ }^{1)}$ Among various isointegral maps they proposed, i.e., QRS, ST-T, QRST, STsegment and Q-zone maps, a QRST isointegral map is interesting because the time integration over the entire QRST complex has been considered to

From *the First Department of Internal Medicine, Yamagata University School of Medicine, Yamagata and **the Department of Internal Medicine, Fujita Gakuen University School of Medicine, Toyoake.

Address for reprint: Isao Kubota, M.D., First Department of Internal Medicine, Yamagata University School of Medicine, Aza-Nishinomae, Zao-Iida, Yamagata-shi, Yamagata 990-23, Japan.

Received for publication July 18, 1983. 
reflect the primary ventricular recovery properties. ${ }^{2)}$ Even in the presence of abnormal ventricular activation as occurs with intraventricular conduction disorders and ectopic origin of excitation, a QRST isointegral map may accurately reflect the ventricular recovery properties. ${ }^{3)}$ In the case of myocardial infarction, the ventricular recovery property was considered to be altered due to a loss of the electromotive force of the infarcted myocardium. Thus, it may be possible to evaluate the site of myocardial infarction by means of QRST isointegral maps.

The purpose of this study was to establish the clinical usefulness of QRST isointegral maps to assess the ventricular asynergic site in patients with previous myocardial infarction. This method may be useful if the site of asynergy can be correctly detected even in the presence of intraventricular conduction disturbances. In the present study, we devised a deviation map technique to evaluate the abnormalities of QRST isointegral maps quantitatively. The deviation map was constructed from a deviation index, which expressed the deviation of the QRST time-integral value from the normal time-integral value at each lead site.

\section{Materials and Methods}

Of 250 consecutive patients who underwent diagnostic left ventriculography and coronary arteriography in our hospital, 41 satisfied all of the following criteria and formed the study population. (1) The patient had a history of a previous acute myocardial infarction, but no new infarction within 6 months prior to the cardiac catheterization. The clinical diagnosis was established by a history of typical chest pain, serum enzyme changes and electrocardiographic changes. (2) The patient had left ventricular asynergy proven by left ventriculography performed in the right anterior oblique position. Left ventricular asynergy was defined as akinetic or dyskinetic wall motion. (3) The patient had significant coronary stenosis proven by coronary arteriography. Occlusion of $75 \%$ or more of the luminar diameter of one or more coronary arteries was defined as significant. (4) The patient had no clinical history of unstable angina pectoris within 6 months prior to the cardiac catheterization. (5) The patient had no other cardiovascular disorders such as hypertension, congenital heart disease, myocardial disease or valvular heart disease.

Informed consent was obtained from all subjects.

Cardiac catheterization:

Left ventriculography and coronary arteriography were performed using 
the Judkins' technique. Left ventriculography was performed both in the 30-degree right anterior oblique and in the 60-degree left anterior oblique positions and these ventriculograms were analyzed for the present study according to the reporting system of the American Heart Association ${ }^{4}$ without knowledge of the map data, using only the results of the right anterior oblique studies. For the purpose of this study, segments 1 and 2 (anterobasal and anterolateral segments) were referred as the anterior wall and segments 4 and 5 (posterobasal and diaphragmatic segments) as the inferior wall. Coronary arteriography was performed in multiple projections.

\section{Data recording and processing:}

Body surface mapping was performed using a HPM-5100 system (Chunichi Denshi Co, Nagoya, Japan) within 1 week prior to the cardiac catheterization. Since the localization of the lead points and the procedures for data sampling and processing have been reported previously, ${ }^{5}$ they will be described here only briefly. Eighty-seven unipolar electrocardiograms (ECGs) distributed over the entire thoracic surface (Fig. 1) with Wilson's central terminal as reference, standard 12 lead-ECG and the Frank X, Y, Z ECGs were sampled simultaneously. Then, the stored signals of each ECG were displayed on a graphic terminal (TEKTRONIX 4006-1). If artifacts were detected in any of the signals, data samplings were repeated. After baseline adjustment by linear interpolation, the data were recorded on a cassette tape in digital format. For this study, the flat portion of the PQ segment was selected as the baseline, and the data samplings were done at the resting expiratory level in the supine position. This system had a resolution of $10 \mu \mathrm{V}$, in the dynamic range $\pm 5 \mathrm{mV}$, with a sampling frequency of 250 samples/ second/channel.

To perform further analysis of the data, the ECG signals were processed off-line on a minicomputer (Texas Instruments 980-B) using a system of map analysis programs dcvcloped at our institution. The input of this minicomputcr system was the cassette tape copied by the above mentioned HPM5100 system. The QRST time integral was calculated for each lead as the algebraic sum of all potentials from the QRS onset to $\mathrm{T}$ offset multiplied by the sampling interval. ${ }^{1)}$ QRS onset and $T$ offset were determined from superimposed Frank X, Y, Z leads and spatial magnitude (Fig. 2). In order to measure the QRS duration, QRS offset was also determined in the same way.

Classification of the patients:

The 41 patients were analyzed in 3 groups according to the site of ven- 


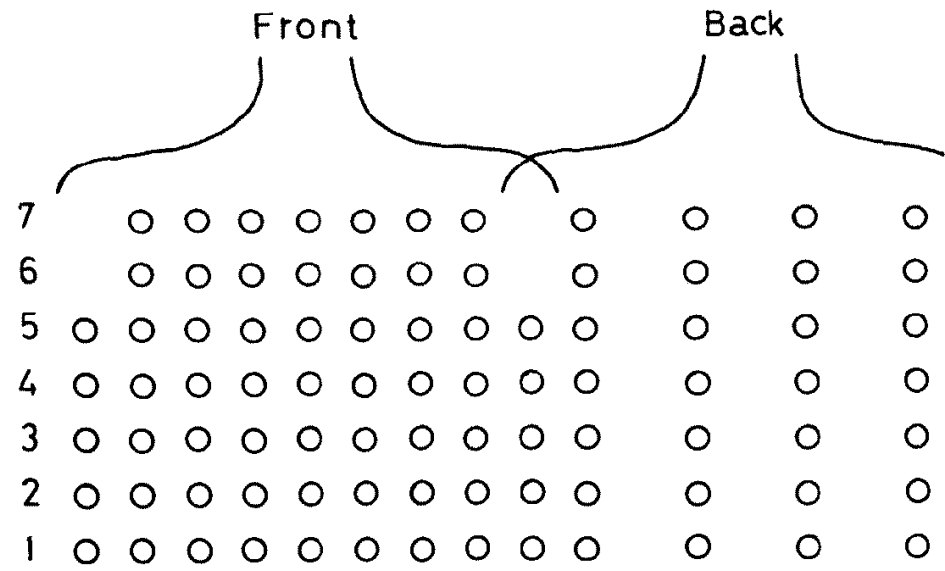
A B C D
D F
G
H I J
K
L $\quad M$

Fig. 1. Electrode sites on the body surface. Eighty-seven lead points were arranged latticelike ( $13 \times 7$ matrix) except for 4 lead points in both midaxillary regions, and covered the entire thoracic surface. Columns $A, E$ and $I$ were positioned in the right midaxillary, midsternal and left midaxillary lines, respectively. Columns B-D and F-H were evenly spaced between columns $A-E$ and $E-I$, respectively. Column $J$ was located so as to make the distance betwcen columns $\mathrm{I}$ and $\mathrm{J}$ equal to that betwcen columns $\mathrm{H}$ and I. Similarly, column $\mathrm{M}$ was located. Columns $\mathrm{K}$ and $\mathrm{L}$ were evenly spaced between columns $\mathrm{J}$ and M. Lead points E6 and E4 were located on the 2 nd and 5 th intercostal spaces, respectively. Row 5 was located equidistant between rows 6 and 4 . Rows 7 and 3-1 were located so as to make the distance between adjacent rows equal.

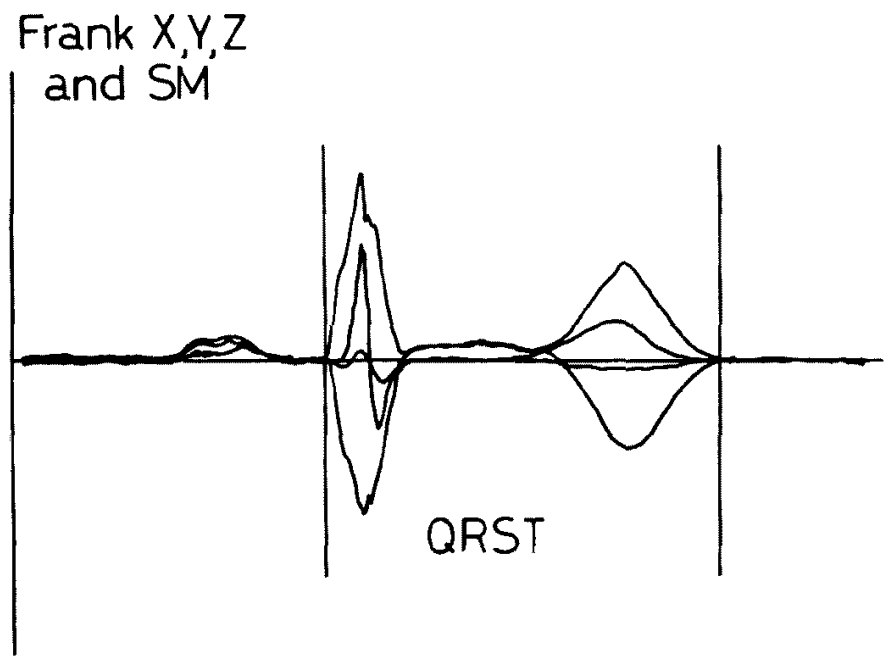

Fig. 2. Superimposed Frank $\mathrm{X}, \mathrm{Y}, \mathrm{Z}$ and spatial magnitude locating the onset of the QRS complex and the offset of the $T$ wave. $S M=$ spatial magnitude. 
tricular asynergy as follows: group ANT consisted of 13 patients with left ventricular asynergy of the anterior wall, group INF consisted of 13 patients with ventricular asynergy of the inferior wall, and group MIX was composed of 15 patients with ventricular asynergy of both the anterior and inferior walls. Of the 41 patients, 2 cases had complete left bundle branch block, 1 had incomplete left bundle branch block, and 1 had complete right bundle branch block. All these 4 cases belonged to group MIX. The other 16 patients had QRS durations longer than $90 \mathrm{msec}$. In order to investigate the influence of the QRS duration on the QRST isointegral map, we divided the patients into 2 subgroups according to the $Q R S$ duration as follows: patients with a QRS duration equal to or less than $90 \mathrm{msec}$ (subgroup A) and patients with a QRS duration greater than $90 \mathrm{msec}$ (subgroup B).

Consequently, the 41 patients were divided into 6 subsets as shown in Table I. In the ANT group, subgroup A contained 6 males and 1 female and subgroup B 6 males. In the INF group, subgroup A contained 6 males and 1 female and subgroup B 6 males. In the MIX group, subgroup A constituted 3 males and 4 females and subgroup B 7 males and 1 female.

\section{Deviation map:}

From the 40 normal males, ages $22-51$ years (mean 33.1 ), the mean value (M) and standard deviation (SD) of the QRST time-integral value at each lead point were obtained. The 40 subjects had no history of cardiovascular disease and all were normal on physical and standard 12 lead-ECG examination. The deviation index (DI) at each lead point was calculated as follows:

$$
\mathrm{DI}=\frac{\mathrm{X}-\mathrm{M}}{\mathrm{SD}}
$$

where X represented the QRST time-integral value at the corresponding lead

Table I. Classification and Clinical Data of the Patients

\begin{tabular}{|c|c|c|c|c|c|c|c|}
\hline \multirow{2}{*}{\multicolumn{2}{|c|}{ Group }} & \multirow{2}{*}{$\mathrm{N}$} & \multirow{2}{*}{$(\mathrm{F})$} & \multicolumn{2}{|c|}{ Age (years) } & \multicolumn{2}{|c|}{ QRS duration (msec) } \\
\hline & & & & Range & Mean & Range & Mean \\
\hline \multirow[t]{2}{*}{ ANT } & A & 7 & (1) & $42-61$ & 52.3 & $76-88$ & 80 \\
\hline & B & 6 & (0) & $42-55$ & 50.3 & $92-108$ & 98 \\
\hline \multirow[t]{2}{*}{ INF } & A & 7 & (1) & $37-69$ & 55.6 & $76-88$ & 80 \\
\hline & B & 6 & $(0)$ & $49-64$ & 54.0 & $92-116$ & 103 \\
\hline \multirow[t]{2}{*}{ MIX } & A & 7 & (4) & $49-68$ & 61.1 & $72-88$ & 80 \\
\hline & B & 8 & (1) & $49-69$ & 57.8 & $96-140$ & 112 \\
\hline \multicolumn{2}{|c|}{ Total } & 41 & (7) & $37-69$ & 55.5 & $72-140$ & 93 \\
\hline
\end{tabular}

Abbreviation: $\mathrm{F}=$ female. 
point of the patient. Deviation maps were constructed by drawing a contour line which connected points of equal DI value. In addition, mean deviation maps were also constructed from the subset mean DI value at each lead site (Fig. 7). In this study we gave attention to the area having a DI of less than -2 and called this area the 'negative deviation area'. Fig. 3 shows the QRST isointegral maps constructed from M+2SD (upper panel), M (middle) and $\mathrm{M}-2 \mathrm{SD}$ (lower) of the normal QRST time-integral value at each lead.

\section{Display :}

The map format for QRST isointegral maps is illustrated in Figs. 3-6 and that for deviation maps in Figs. 4-7. In both formats, each rectangular
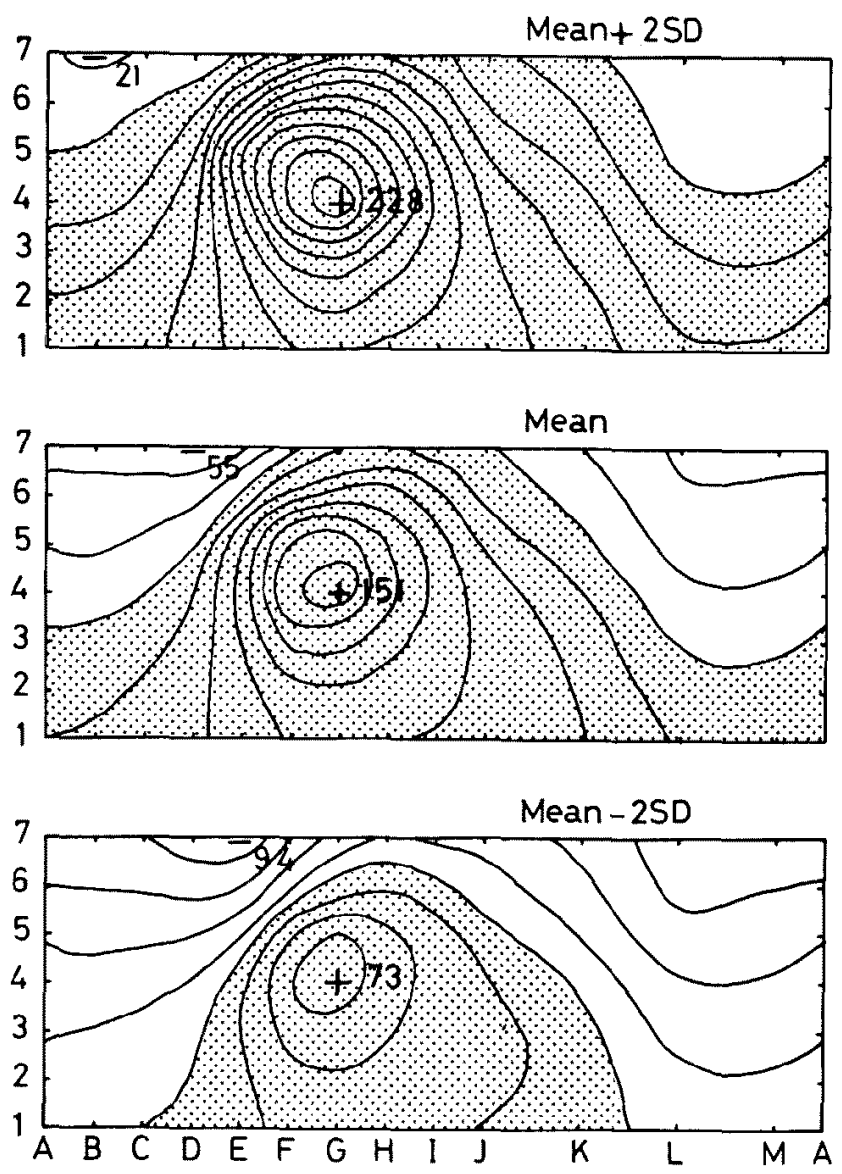

Fig. 3, QRST isointegral maps constructed from mean +2 standard deviations (upper panel), mean (middle panel) and mean-2 standard deviations (lower panel) of normal QRST time-integral value at each lead point. See Methods for description of map-display format. 
area represents the torso, with the left half corresponding to the anterior chest and the right half to the back. Thus, both the left and right margins represent the right midaxillary line. Precise electrode positions on the body surface are described in Fig. 1. In all QRST isointegral maps, contour lines were drawn at intervals of 20 microvolt -seconds, and the dotted region corresponds to the area with the positive time-integral value. The values of maximum and minimum time integrals are displayed in the map. In each deviation map, contour lines are depicted at an interval of one deviation index, and the shaded region represents the negative deviation area, where the deviation index is less than -2 . The value of the minimum deviation index is displayed in the map.

\section{Results}

\section{Patients with anterior asynergy (group $A \mathcal{N T}$ ):}

This group was composed of 7 patients with QRS durations ranging from 76 to $88 \mathrm{msec}$ (mean 80, subgroup A) and 6 patients with QRS durations ranging from 92 to $108 \mathrm{msec}$ (mean 98, subgroup B). QRST isointegral maps and deviation maps in this group were consistent in all subjects. Fig. 4A depicts the QRST isointegral map (upper panel) and deviation map (lower panel) of a patient with a QRS duration of $88 \mathrm{msec}$ in subgroup A, and Fig. 4B shows those of a patient with a QRS duration of $108 \mathrm{msec}$ in subgroup B. In both QRST isointegral maps, negative time-integral values occupied the area over the left upper anterior chest unlike a normal map (Fig. 3, middle panel) and produced an indentation of the positive timeintegral area. This indentation of the positive area from the left upper anterior chest was characteristic of the QRST isointegral maps in this group. Furthermore, in deviation maps of this group, the negative deviation areas were consistently observed around the left middle and upper anterior chest as shown in the lower panels of Fig. 4.

\section{Patients with inferior asynergy (group INF):}

This group was composed of 7 patients whose QRS durations ranged from 76 to $88 \mathrm{msec}$ (mean 80, subgroup A) and 6 patients with QRS durations that ranged from 92 to $116 \mathrm{msec}$ (mean 103, subgroup B). Fig. 5A shows the QRST isointegral map (upper panel) and deviation map (lower panel) of a patient in subgroup A whose QRS duration was $84 \mathrm{msec}$, and Fig. $5 \mathrm{~B}$ depicts those of a case in subgroup B with a QRS duration of $112 \mathrm{msec}$. In both QRST isointegral maps, negative time-integral values extended to the lower right anterior chest and lower back compared with a normal map 
(A) T. O. 49 yrs male

QRS duration $88 \mathrm{msec}$
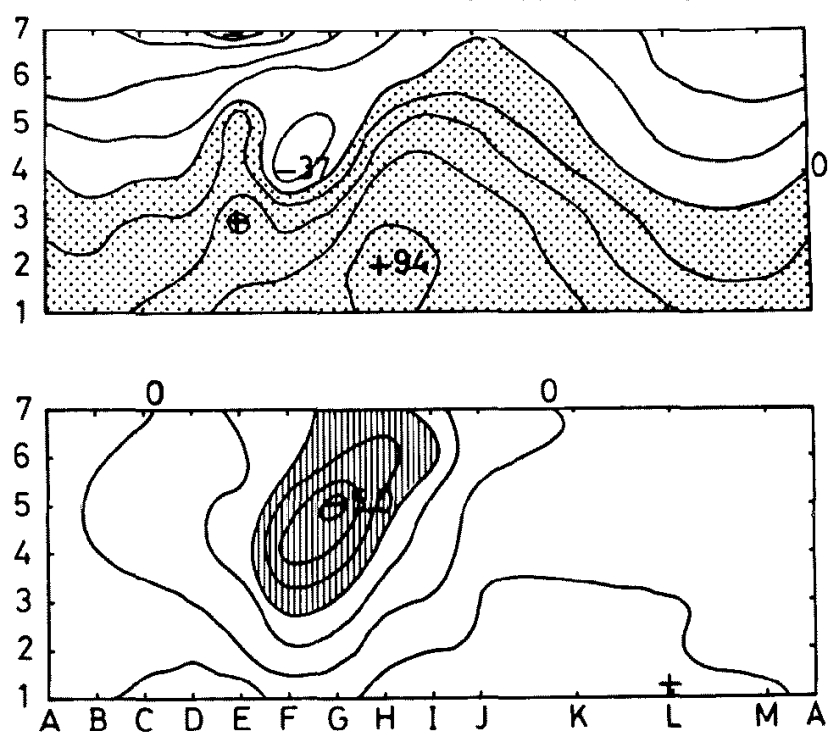

(B) T. T. 42 yrs male

QRS duration $108 \mathrm{msec}$
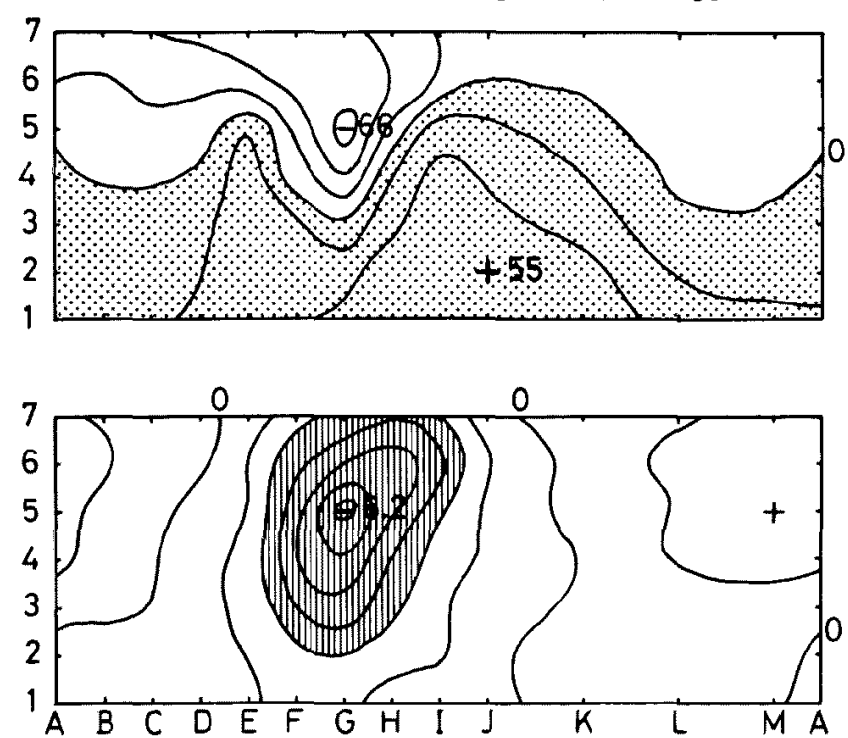

Fig. 4. QRST isointegral maps (upper panels) and deviation maps (lower pancls) of 2 representativc cases in group ANT; (A) shows those of the patient in subgroup $A$, while (B) depicts those in subgroup B. QRST isointegral maps and deviation maps in these 2 cases resembled each other. In both QRST isointegral maps, an indentation of the positive time-integral area from the left upper anterior chest was observed. Note that these 2 patients both had negative deviation areas around the middle and upper portions of the left anterior chest. Sec Methods for detailed description of map-display format. 
(A) Y.N. 69 yrs temale
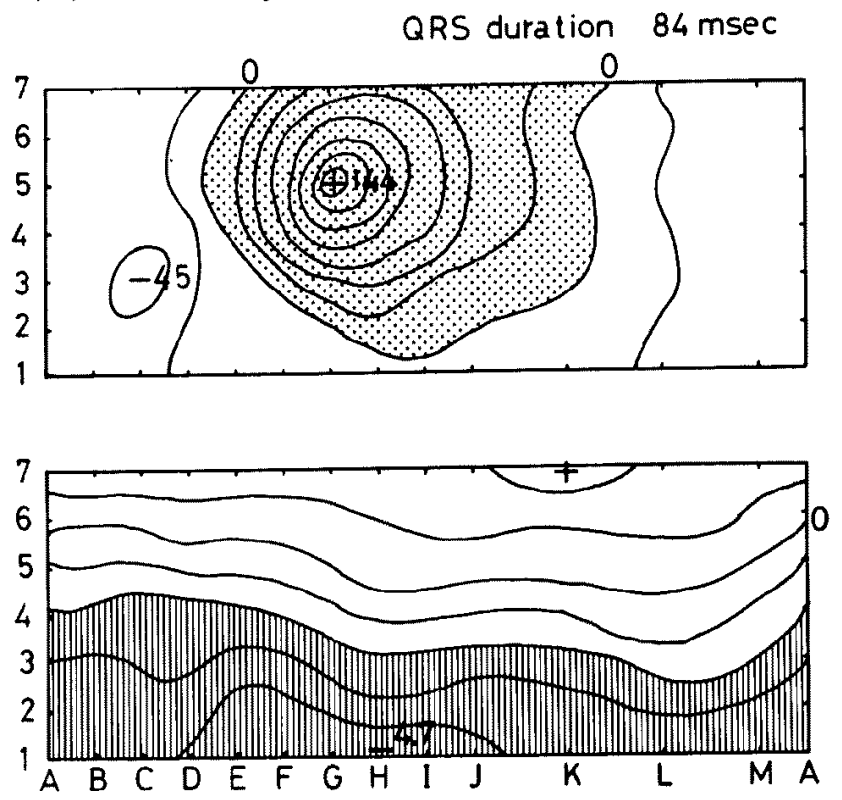

(B) S. 1. 53 yrs male
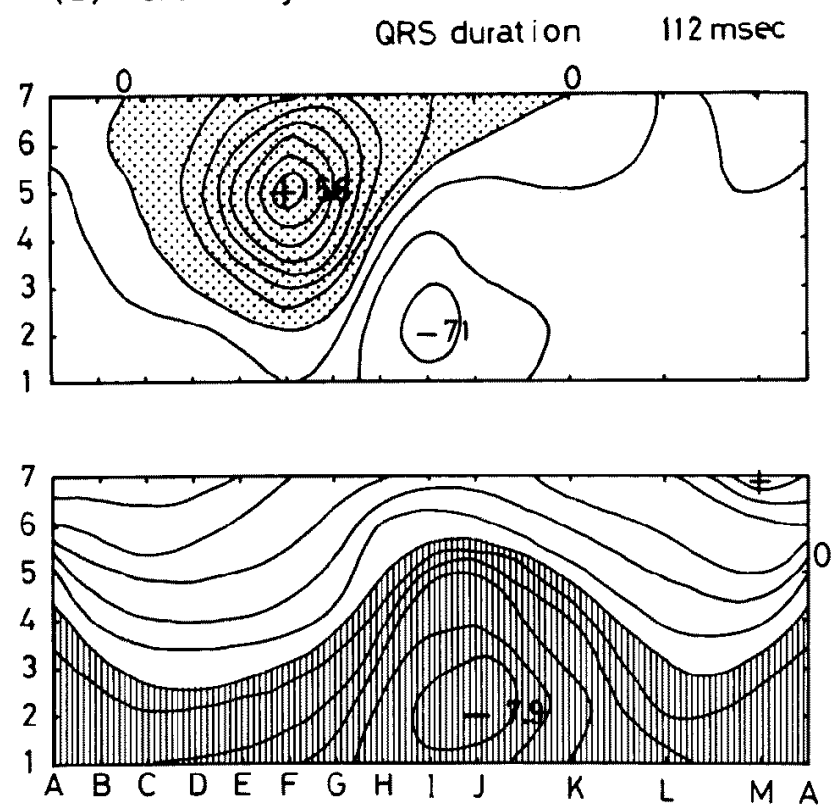

Fig. 5. QRST isointegral maps (upper panels) and deviation maps (lower panels) of 2 cases in group INF. (A) shows those of the patient in subgroup $A$ and (B) depicts those of the case in subgroup $B$. These 2 patients had similar patterns in both QRST isointegral maps and deviation maps. In the QRST isointegral maps, the positive time-integral values were located over the left anterior chest and negative values over the right anterior chest and the back. Note that the negative deviation areas were observed spreading around the inferior portion of the thoracic surface. See Methods for detailed description of map-display format. 
(Fig. 3, middle panel). Thus, the positive values were located mainly over the left upper anterior chest. This distribution of positive and negative timeintegral values was consistently observed in this group. Deviation maps in this group were also characterized by the site of the negative deviation area. All patients in this group had the negative deviation areas spreading over the inferior portion of the thoracic surface as shown in Fig. 5.

Patients with both anterior and inferior asynergies (group $M I X)$ :

This group consisted of 7 patients with QRS durations ranging from 72 to $88 \mathrm{msec}$ (mean 80 , subgroup A) and 8 patients with QRS durations of from 96 to 140 msec (mean 112, subgroup B). Fig. 6A represents the QRST isointegral map (upper panel) and deviation map (lower panel) of 1 patient belonging to subgroup A whose QRS duration was $88 \mathrm{msec}$, while Fig. $6 \mathrm{~B}$ shows those of 1 patient in subgroup B with a QRS duration of $124 \mathrm{msec}$. Apparently, these two QRST isointegral maps present no similarities; one map of the patient in subgroup $A$ had a positive time-integral area around the inferior portion of the chest, whereas a negative time-integral area was observed in the same portion in another map of the case in subgroup B. In this group, no consistent positive and negative time-integral distribution pattern was observed in the QRST isointegral maps although all these patterns were quite different from the normal map (Fig. 3, middle). Even if QRST isointegral maps of the 2 subgroups were examined separately, we could not obtain any common distribution pattern for either subgroup.

On the other hand, both deviation maps in Fig. 6 show quite similar patterns; the negative deviation areas are visible around the middle and inferior portions of the left anterior chest. The case presented in Fig. 6B had complete left bundle branch block. Including this case, 2 patients with complete left bundle branch block, 1 with incomplete left bundle branch block and 1 with complete right bundle branch block comprised subgroup B of the group MIX. Nevertheless, all deviation maps of this group had a consistent pattern that was characterized by the appearance of the negative deviation area around the middle and inferior portions of the left anterior chest as shown in Fig. 6.

\section{Summary of deviation maps:}

Fig. 7 shows mean deviation maps constructed from the subset mean deviation index at each electrode site. Since there were 3 groups (groups ANT, INF and MIX) and each group had 2 subgroups (subgroups A and B), six different mean deviation maps were constructed. In the figure, mean deviation maps of group ANT are displayed in upper panels, maps of group 
(A) Y.G. 49 yrs male

QRS duration $88 \mathrm{msec}$
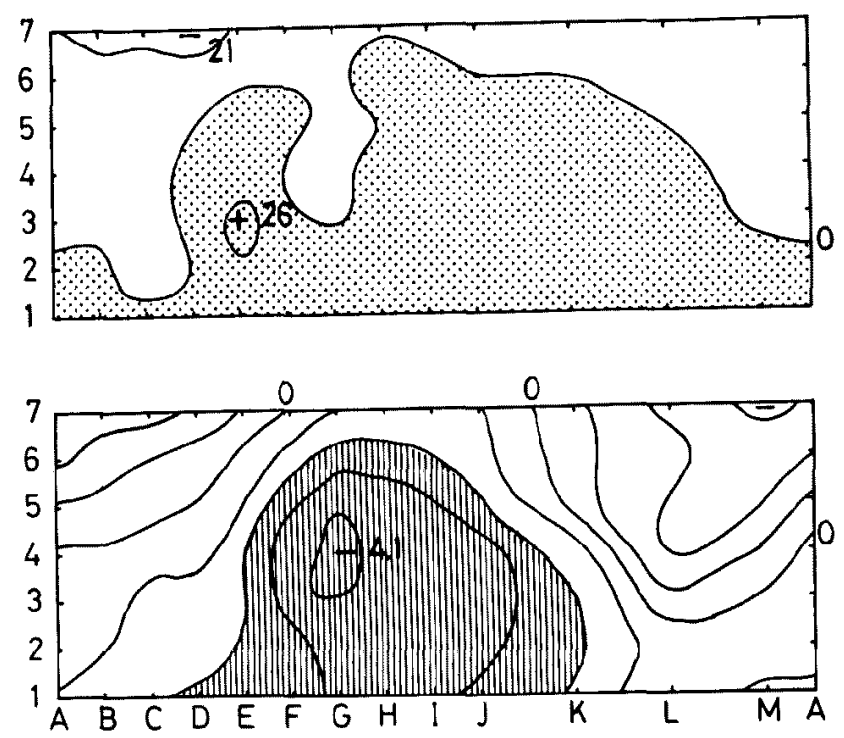

(B) G.S. 60 yrs male

QRS duration $124 \mathrm{msec}$
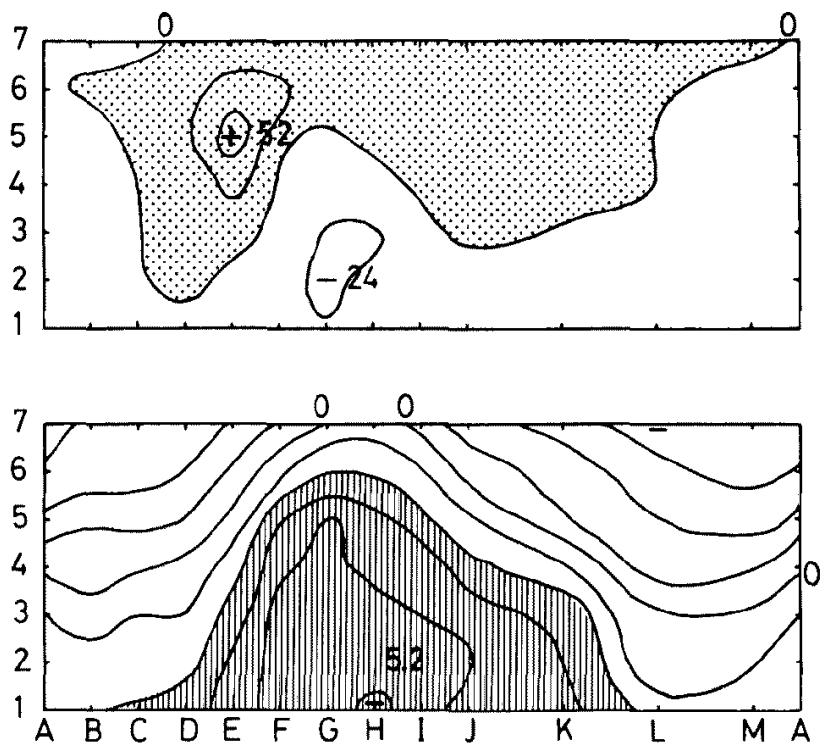

Fig. 6. QRST isointegral maps (upper panels) and deviation maps (lower panels) of 2 cases in group MIX. (A) represents those of the patient belonging to subgroup $A$, while (B) shows those of the case in subgroup $B$. Apparently, these two QRST isointegral maps present no similarities. On the other hand, the deviation maps show quite similar patterns. Note that the negative deviation areas are visible around the middle and inferior portions of the left anterior chest. See Methods for detailed description of map-display format. 
(A)

(i) ANT

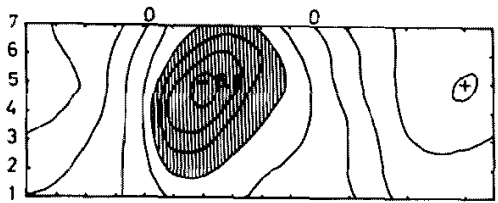

(2) INF

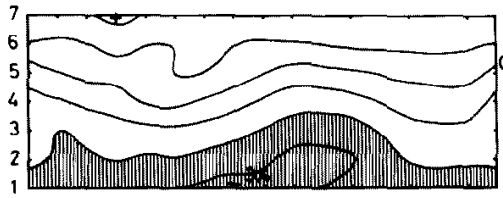

(3) $\mathrm{MIX}$

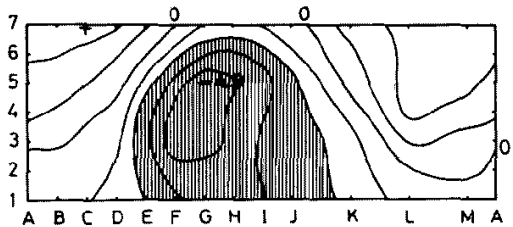

(8)
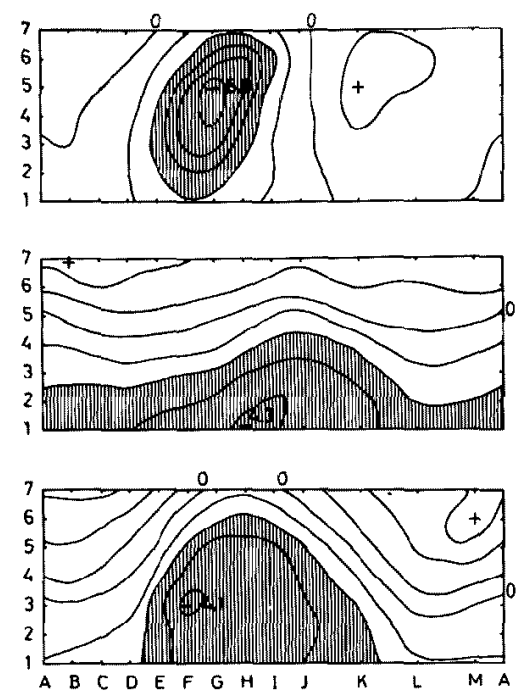

Fig. 7. Mean deviation maps constructed from the subset mean deviation index value at each electrode site; maps of group ANT are displayed in the upper panels, maps of group INF in the middle panels and those of group MIX are depicted in the lower panels. In addition, the left panels represent the maps of subgroup $A$ and the right panels show those of subgroup $B$. Note that the mean deviation maps of each group are easily distinguished by the characteristic distribution patterns of the negative deviation areas, and that maps of the 2 subgroups in each group closely resemble each other. See Methods for detailed description of map-display format.

INF in the middle panels and those of group MIX are depicted in the lower panels. In addition, the left panels of the figure represent the maps of subgroup A and right panels those of subgroup B. Note that the mean deviation maps of each group are easily distinguished by means of the distribution pattern of the negative deviation areas, and that maps of the 2 subgroups in each group closely resemble each other. A characteristic distribution pattern of the negative deviation area was found in each group: group ANT had the negative deviation area around the middle and upper portions of the left anterior chest, group INF had it spreading over the inferior portion of the thoracic surface and group MIX possessed it around middle and inferior portions of the left anterior chest.

\section{Discussion}

This study demonstrated that QRST isointegral maps of patients with myocardial infarction provide information about the site of left ventricular 
asynergy regardless of the QRS duration. The distinctive patterns of QRST isointegral maps were recognized in both patients with anterior asynergy (group ANT, Fig. 4) and those with inferior asynergy (group INF, Fig. 5). On the other hand, we could not detect any consistent distribution pattern of positive and negative time-integral values in the original QRST isointegral maps of patients with both anterior and inferior asynergies (group MIX, Fig. 6). To evaluate the abnormalities of QRST isointegral maps quantitatively, we devised a deviation map technique. By using deviation maps, we were able to find characteristic patterns not only in groups ANT and INF, but also in group MIX. The negative deviation area was always found around the middle and upper portions of the left anterior chest in patients with anterior asynergy (group ANT, Fig. 4), while it was invariably observed around the lower thoracic surface in those with inferior asynergy (group INF, Fig. 5). As might be expected from these results, all patients with both anterior and inferior asynergies (group MIX) had the negative deviation areas around middle and inferior portions of the left anterior chest (Fig. 6). Thus, the ventricular asynergic site was correctly diagnosed by means of the location of the negative deviation area even if the patient had an intraventricular conduction disturbance (Fig. 7). The independency of QRS durations was compatible with the theory that the QRST time-integral value reflects the intrinsic ventricular recovery properties. It was suggested that the negative deviation areas in our study could reveal on the body surface the abnormally altered intrinsic ventricular recovery properties resulting from a loss of the electromotive force of the infarcted myocardium.

In the assessment of myocardial infarction, QRST isointegral maps have two advantages compared with isopotential maps recorded during the QRS period. The first is the ability to manage those patients with longer QRS durations such as occur in intraventricular conduction disturbances as well as those without them. If the activation sequence was changed or prolonged by the occurrence of a myocardial infarction, the serial isopotential maps recorded during the QRS period are likely to be affected by conduction abnormalities in addition to loss of the electromotive forces due to the myocardial infarction. The qualitative and quantitative evidence that the QRST time-integral value in extensively sampled body surface ECGs and cardiac surface electrocardiograms is largely independent of the ventricular activation sequence has been reported by Abildskov et al. ${ }^{3,61,7)}$

The second advantage is the conciseness of the data expression. A major problem with body surface mapping is the difficulty in developing a practical means of analyzing and displaying the enormous quantity of data. ${ }^{1}$ Although the isopotential maps allow detailed analysis of sequential changes 
in ECG potentials throughout the cardiac cycle, the enormous data mass makes display cumbersome and the assessment of serial recordings timeconsuming. On the contrary, the QRST isointegral maps generated in this study offer a rapid means of identifing the ventricular asynergic site in patients with myocardial infarction.

We conclude from this study that the QRST isointegral maps, especially their deviation maps, are a practical method of identifying the ventricular asynergic site in patients with myocardial infarction. The independency of QRS duration is important because this characteristic is not present in isopotential maps or conventional electrocardiograms.

\section{ACKNOWLEDGMENTS}

The authors express their thanks to Professor Kozui Miyazawa for his valuable help and advice in this study.

\section{References}

1. Montague TJ, Smith ER, Cameron DA, Rautaharju PM, Klassen GA, Flemington CS, Horacek $B M$ : Isointegral analysis of body surface maps. Surface distribution and temporal variability in normal subjects. Circulation 63: 1166, 1981

2. Wilson FN, MacLeod AG, Barker PS, Johnston FD: The determination and the significance of the ventricular deflections of the electrocardiogram. Am Heart J 10: 46, 1934

3. Lux RL, Urie PM, Burgess MJ, Abildskov JA: Variability of the body surface distributions of QRS, ST-T and QRST defection areas with varied activation sequence in dogs. Cardiovasc Res 14: 607,1980

4. Austin WG, Edward JE, Erve RL, Gensini GG, Gott VL, Griffith LSC, McGoon DC, Murphy ML, Roe BB: AHA Committee Report: A reporting system on patients evaluated for coronary artery disease. Circulation 51: 5, 1975

5. Watanabe T, Toyama J, Toyoshima H, Oguri H, Ohno M, Ohta T, Okajima M, Naito Y, Yamada K: A practical microcomputer based mapping system for body surface, precordium, and epicardium. Comput Biomed Res 14: 341, 1981

6. Abildskov JA: Effects of activation sequence on the local recovery of ventricular excitability in the dog. Circulat Res 38: 240, 1976

7. Urie PM, Burgess MJ, Lux RL, Wyatt RF, Abildskov JA: The electrocardiographic recognition of cardiac states at high risk of ventricular arrhythmias. Circulat Res 42:350, 1978 